\title{
Biosynthesis and Modulation of Terpenoid Indole Alkaloids in Catharanthus roseus: A Review of Targeting Genes and Secondary Metabolites
}

\author{
Mukesh Kumar Sharma1,2* (D), Mohan Kumar ${ }^{1}$ and Renu ${ }^{1}$ \\ ${ }^{1}$ Department of Biotechnology, Maharaj Vinayak Global University, Jaipur - 302 028, Rajasthan, India. \\ ${ }^{2}$ Department of Botany, Vishwa Bharti PG College, Sikar - 332 001, Rajasthan, India.
}

\begin{abstract}
The medicinal plant $C$. roseus synthesizes biologically active alkaloids via the terpenoid indole alkaloid (TIAs) biosynthetic pathway. Most of these alkaloids have high therapeutic value, such as vinblastine and vincristine. Plant signaling components, plant hormones, precursors, growth hormones, prenylated proteins, and transcriptomic factors regulate the complex networks of TIA biosynthesis. For many years, researchers have been evaluating the scientific value of the TIA biosynthetic pathway and its potential in commercial applications for market opportunities. Metabolic engineering has revealed the major blocks in metabolic pathways regulated at the molecular level, unknown structures, metabolites, genes, enzyme expression, and regulatory genes. Conceptually, this information is necessary to create transgenic plants and microorganisms for the commercial production of high-value dimer alkaloids, such as vinca alkaloids, vinblastine, and vincristine In this review, we present current knowledge of the regulatory mechanisms of these components in the $C$. roseus TIA pathway, from genes to metabolites.
\end{abstract}

Keywords: Metabolites,Vinblastine and Vincristine, Plant signalling components, Terpenoid indole alkaloids, Transcriptomic factors

\footnotetext{
*Correspondence: mukeshsharma.dt@gmail.com

(Received: August 27, 2021; accepted: September 09, 2021)

Citation: Sharma MK, Kumar M, Renu. Biosynthesis and Modulation of Terpenoid Indole Alkaloids in Catharanthus roseus: A Review of Targeting Genes and Secondary Metabolites. J Pure App/ Microbiol. 2021;15(4):1745-1758. doi: 10.22207/JPAM.15.4.05

(C) The Author(s) 2021. Open Access. This article is distributed under the terms of the Creative Commons Attribution 4.0 International License which permits unrestricted use, sharing, distribution, and reproduction in any medium, provided you give appropriate credit to the original author(s) and the source, provide a link to the Creative Commons license, and indicate if changes were made.
} 
TIAs are the main producers of alkaloids in plants, with over 3000 alkaloids discovered to date. Alkaloids are nitrogen-containing natural products that are mostly derived from amino acids, including Phe, Tyr, Trp, Lys, and Orn. ${ }^{1}$ More than 50 biosynthetic events, composed of genes, enzymes, regulators, and intra-/intercellular transporters, occur in the biosynthesis of TIAs in C. roseus. ${ }^{2}$ The common precursor for all TIAs is strictosidine, which can be converted into a series of monoterpenoid indole alkaloids (MIAs) with diverse structures and biological functions. ${ }^{1}$ Strictosidine is an essential intermediate derived from the condensation reaction of secologanin with tryptamine, catalyzed by strictosidine synthase (STR) (EC 4.3.3.2), ${ }^{3}$ in alkaloid biosynthesis and is an important step in TIAs biosynthesis. ${ }^{4}$ Strictosidine, which is comprised of tryptamine and a $\mathrm{C}^{10}$ component made up of iridoid secologanin. Several bioactive compounds are biologically important in animals. However, these are only found in eight plant species and the most important are Apocynaceae, the dogbane family (order Gentianales) of flowering plants, Loganiaceae family (order Gentianales), and Rubiaceae or coffee, madder, or bedstraw family. However, these are only found in eight plant species (the most important of which are Apocynaceae, Loganiaceae, and Rubiaceae). Some TIAs have been used to fabricate medicines, including the anti-malarial quinine isolated from Cinchona officinalis, the antineoplastic (camptothecin) from Camptotheca acuminate, homeopathic medicine (strychnine) from Strychnosnux-vomica, reserpine from Rauvolfia species, and the anticancer agents, vinblastine and vincristine, from Cataranthusroseus. ${ }^{5}$

Cataranthusroseus (C. roseus) is considered a pharmaceutical factory and is associated with a high cost owing to its broad range of applications. C. roseus $(>130)$ is an essential biosynthesis plant in the pharmaceutical industry. In addition to the most popular bisindole alkaloids, ajmalicine, an antihypertensive, and serpentine, a sedative (vinblastine and vincristine) are derived from $C$. roseus. The high price of these TIAs is caused by the extremely low content of dimer alkaloids in C. roseus and the difficulty associated with their extraction and purification. These two dimer vinca alkaloids are formed by the coupling of catharanthine (a derivative of strictosidine) and vindoline. ${ }^{6}$ Vinblistine and vincristine act as anticancer alkaloids, which are well explained by their metabolic engineering effects. ${ }^{76}$ Although comprehensive chemical synthesis of these complex alkaloids is of research interest, owing to their low yields, they are unlikely to be used economically. The hydrophobic reaction of the vindoline and catharanthine combination, which causes precipitation owing to the peroxidase compounds in the plant species, has been biologically replicated and is currently used to combine more polymers. An in vitro production method was developed based on plant cell cultivation practices or using $C$. roseus hair cultivation roots. However, vindoline, which is one of the substrates for bisindole alkaloids, was not produced using this method. Investigators must completely understand the gene, biomolecular enzymes, and intermediate and regulatory mechanisms of the TIA biosynthesis pathway to develop new sources of bioactive molecules (Fig. 1).

\section{C. roseus and secondary metabolites}

C. roseus is an effective herbal medicine that has a family of secondary metabolites containing terpenoid indole alkaloids (TIAs). More than 130 TIAs are known to be biosynthesized. ${ }^{7}$ Owing to their therapeutic abilities, the serpentine, ajmalicine, and the bisindole alkaloids, vinblastine, vincristine, and -3,4-anhydrovinblastine, were assessed for several decades, which led to their commercialization (serpentine, ajmalicine, and the bisindole alkaloids vinblastine, vincristine, and -3,4-anhydrovinblastine). Vincristine and vinblastine are two approved alkaloids for the treatment of a variety of cancers. ${ }^{7}$ Because the quantity of these plant metabolites is very small, it is difficult to isolate them..$^{8,9}$ As behavioral challenges, such as climate change, pests, and geographical and seasonal boundaries, remain unaffected, plant tissue harvesting is considered an alternative approach for the biosynthesis of bioactive molecules. Owing to regulatory restrictions, alkaloid production in undifferentiated cells is very low or possibly non-existent, which is clearly a hindrance to commercial-scale manufacturing. ${ }^{10}$ Currently, metabolic engineering is considered a possible remedy to avoid the major controlling issues. Overexpression of heterologous or endogenous genes encoding rate-limiting 
processes, suppression of competitive pathways, or overexpression of regulatory transcription factors are possible solutions for bypassing bottlenecks.

Plant metabolism is a complex network that produces metabolites through metabolic pathways. The metabolome is not only the outcome of its genome and proteome, but also a biological response to its surroundings. People benefit from plant metabolic products because plants produce an extensive range of fascinating and beneficial molecules, including dietary supplements, pigments, pesticides, fragrances, and medications. Important efforts have been made to study the regulation of plant secondary metabolism to increase the convenience of these biologically active compounds for human use. Hormonal elicitation, precursor feeding, oxidative stress, and genetic alterations have been employed to modify critical metabolite processes in plants. However, routes do not function in isolation and must always be interconnected with other pathways that are metabolically very active. Engineering used in the production of metabolites can have an impact on other pathways; therefore, changing just one pathway is not always the best option.

Advanced analysis regarding the biosynthesis route and its regulation significantly promotes successful metabolic engineering. Many enzymatic stages in TIA biosynthesis have been scrutinized and studied. ${ }^{1}$ The accessibility of recombinant technologies with genomics and metabolomics techniques have encouraged the development of the metabolic engineering MIAs pathway. ${ }^{11}$ To the best of our knowledge, many enzymatic stages of TIA biosynthesis remain unknown. Main transcription activators and repressors (Table 1 ) regulate primary metabolism in the TIA pathway. ORCA3 is a master regulator of the release of cytokines involved in primary metabolism as well as the TIA pathway, but not all of them. As a result, other unidentified regulatory proteins that influence various groups of genes in the pathway must exist. Identifying additional transcription factors, enzymatic processes, and their associated genes will thus allow us to better understand the TIA pathway and may allow us to modify plant cells to generate necessary alkaloids in high quantities in an industrial production system.

Several investigations on alkaloidproducing plants have revealed that alkaloid production and accumulation are tightly controlled processes. ${ }^{12}$ MIAs are abundant in active metabolite plants, making them a rich source of medicinal therapies. Alkaloids such as antineoplastics (vinblastine and vincristine), antihypertensives (ajmalicine and ajmaline), and antimalarial (quinine) belong to this class. To draw conclusions from the large amounts of data obtained using high genomics technology, advanced bioinformatics tools are necessary. Studies combining transcriptomes and expression profiling have enabled assessments of the synthesis of plant-derived medicines in C. roseus ${ }^{13-15}$ as well as a better understanding of plant metabolic activities.

\section{Essential Components of Tia Biosynthesis}

In C. roseus, TIA biosynthesis, which is comprised of at least 30 coordinated enzyme steps, produces at least 35 recognized intermediate enzymes. The pathway requires at least four cellular components and at least five major subcellular compartments in each of these cells, implying that transport is a substantial rate key factor involving, among other things, physiochemical properties, and various types of active selective proteins that are transported from cells to other areas. ${ }^{16}$ Thirty biosynthetic genes and four different types of target proteins have been cloned and discovered to date. Unfortunately, there are still unresolved aspects of TIA biosynthesis. In $C$. roseus, alkaloids are formed via the combination of two principal metabolic processes, namely the shikimate and secoiridoid processes, which contribute the indole and terpene moieties to the essential backbone of strictosidine. ${ }^{7,17}$ Metabolic engineering is a technique that uses gene transfer technologies to alter metabolic pathways and metabolite production. With an increase in the understanding of the TIA pathway and its metabolic genes, metabolic engineering of TIA biosynthesis in $C$. roseus is being used to increase the yields of selected TIAs.

TIAs in $C$. roseus have been the focus of excellent contributions and extensive indepth studies in different categories. The genes, 
enzyme(s), transcription factors, transmediate pathways, and divisions of TIA production in C. roseus cell cultures, hairy roots, and plants have been assessed using omics technologies, such as genome, transcriptomics, proteomics, and metabolism. These technologies will be extremely useful for elucidating most of the unsolved sections of the iridoid pathway, such as catharanthine production, transport, and signaling, and control by transcription factors, such as the octadecanoid-responsive Catharanthus APATELA2-domain. ORCAs

Biosynthesis of alkaloid and its role in $C$. roseus

In C. roseus, TIA biosynthesis, which is comprised of at least 30 coordinated enzyme steps, produces at least thirty-five known precursors proteins. The pathway requires at least four cellular components and at least five major subcellular compartments in each of these cells, implying that transport is a substantial rate key factor involving, among other things, physiochemical principles and various types of active selective transporter proteins. ${ }^{16}$ Thirty biosynthetic genes and four different types of target proteins have been cloned and discovered to date. Unfortunately, there are still unsolved aspects of TIA biosynthesis. In $C$. roseus, alkaloids are formed via the combination of two principal components that are involved in metabolic processes, namely the shikimate and secoiridoid pathways, which contribute the indole and terpene moieties to the essential backbone of strictosidine. ${ }^{7,17}$

The Biosynthetic Pathways for Shikimatechorismate-indole and its localization

The shikimate pathway, which begins with phosphoenolpyruvate and erythrose-4-phosphate and concludes with chorismite, ${ }^{18}$ is a significant metabolic pathway for both primary and secondary metabolism. Anthranilate synthase (AS) on the branch leading to tryptophan; chorismate mutase (CM) upon its branch leads to phenylalanine and tyrosine; isochorismate synthase (ICS) on the branch leads to isochorismate, salicylates, and 2,3dihydrobenzoic acid (2.3dihydrobenzoic acid (2,3dihydrobenzo)). ${ }^{19}$ The aromatic tryptophan is formed by the anthranilate route, which provides the indole moiety for TIAs (Fig. 2). The first major protein that is mainly used in the production of tryptophan and indole-3-acetic acid, AS, is composed of two large (-subunits) and two small (-subunits) subunits. ${ }^{20}$ The -subunit of AS catalyzes the conversion of chorismate to anthranilate and is subject to feedback inhibition mediated by tryptophan. ${ }^{21,22}$ The tryptophan route is assumed to begin in plastids. ${ }^{23}$ As the TDC enzyme primarily functions in the cytoplasm, tryptophan must exit plastids and enter the cytosol, where it is decarboxylated by TDC to yield tryptamine, which is then moved to the cell vacuole and condensed with secologanin. The phenylpropanoid pathway genes, $P A L, C 4 H$, and $C H S$, are expressed in the leaf epidermis of $C$. roseus. ${ }^{24,25}$

The MEP-secoiridoid pathway and its localization

TIA terpenoids are derived in part from secologanin, which is produced via the secoiridoid pathway, and geranyl pyrophosphate, a universal monoterpene precursor (GPP). Isopentenyl diphosphate (IPP) is the basic precursor of all isoprenoids and is produced in plants via two different approaches. Approach one is the mevalonate pathway, which produces triterpenes (sterols) and some sesquiterpenes. ${ }^{26,27}$ The MEP pathway has seven enzymatic steps which begins from the condensation of pyruvate and glyceraldehyde 3 phosphate to the end of IPP synthesis. ${ }^{28}$ The first step of the MEP pathway is catalyzed by 1 deoxy Dxylulose 5 phosphate synthase (DXS), which catalyzes the condensation of pyruvate and glyceraldehyde 3 phosphate to 1 deoxy Dxylulose 5 phosphate. The enzyme, 1 deoxy Dxylulose 5 phosphate reductoisomerase, converts this product to 2 Cmethyl Deritriol 4 phosphate (DXR). The final IPP product is converted to dimethylallyl diphosphate (DMAPP) by isopentenyl diphosphate isomerase (IDI). The coding gene has been cloned from many channels, such as CrIDI1. IPPI is the abbreviated name of this enzyme in all tissues. CrIDI1 produces short- and long-term transcripts, resulting in an $\mathrm{N}$-terminal transit peptide (TP), with or without protein. ${ }^{29}$ GPP synthase (GPPS) condenses IPP and DMAPP to produce geranyl diphosphate (GPP), which is the entry point for the production of monoterpenoids. It is the key step in monoterpenoid branching to the TIA pathway after IPP is produced. The enzyme belongs to the short-chain prenyltransferase family through the MEP pathway. ${ }^{30}$ The protein sequences encoded by the three genes from $C$. roseus were 
found to be similar to the large and small subunits of heteromeric GPPS and homopolymeric GPPS. ${ }^{31}$ CrGPPS. LSU is a diterpene enzyme that produces GPP and geranyl diphosphate (GGPP). CrGPPS. SSU can be used as the main regulator of TIA biosynthesis to a certain extent, and CrGPPS is the homopolymerase that forms GPP. CrGPPS. LSU and heteromeric CrGPPS.LSU are stable under normal conditions. LSU/CrGPPS is a combination of LSU and CrGPPS. In C. roseus, SSU provides a GPP reference level for TIA production. The enhanced expression of SSU under biotic and abiotic stresses can increase GPPS activity through the interaction of LSU and SSU, thereby promoting the GPP cluster of TIA. The secoiridoid pathway begins with the formation of geraniol and ends with the production of secologanin. In 2011, only three well-formed stages (including enzymes and linked genes) were found. One enzyme is cytochrome geraniol 10-hydroxylase (P450), also known as geraniol 8- oxidase, which is responsible for the hydroxylation of geraniol to 8-hydroxygeraniol. ${ }^{32,33}$ One of the other two enzymes participate in the iridoid pathway. Maleic acid methyltransferase (LAMT) ${ }^{25}$ catalyzes the methylation of loganin and cytochromes, which catalyze the conversion of loganin in the synthesis of seroganin. P450 (CYP). ${ }^{35,36}$ The final enzyme is ganic acid synthase. The geraniol synthase (GES) enzyme ${ }^{37}$ has been cloned, including in C. roseus, and is a member of the terpene synthase and iris synthase (IS) family; it is a type of short-chain oxidoreductase-recruited cyclase. ${ }^{38}$ Purified recombinant GES protein was used to prepare a catalyst for GPP conformation in GPP, and the km value for GPP was $58.5 \mu \mathrm{M}$. The role of the IS enzyme is to circulate in the iris of eight geraniums, possibly combining the preliminary NAD $(\mathrm{P}) \mathrm{H}$-dependent reduction step with the additives from Diels Alder to Michael. ${ }^{38}$ Importantly, the remaining four stages of enzymes have been discovered, and their coding genes have been cloned..$^{39-41}$ In the presence of NAD + , the enzymes 8-hydroxygeraniol, 8-hydroxygeraniol, and 8-oxygeraniol after $\mathrm{G} 80$ (8HGO) can be oxidized to substrates 8-hydroxygeraniol and 8-hydroxygeraniol. The aldehyde group on alcohol and 8-oxygeranal is used for the 8-catalytic enzyme conversion of CYPgalo7 oxidase to oxidize acid (7DLGT), which uses glucose UDP as a sugar donor to form 7-deoxypolysaccharide acid, which converts the enzyme iridial into 7-deoxypolysaccharide acid. The final step in the formation of the loganin backbone protein is catalyzed by the production of loganic acid (7DLH) 7deoxyhydroxylase, which is also linked to the almost identical P450 subfamily of SLS. ${ }^{40}$ These results enabled the full characterization of the secoiridoid pathway. Clarification clearly shows the progress in the elucidation of biosynthesis. First, purification of the enzyme and sequence analysis of a part of the AA sequence were performed. Accordingly, the metabolites of each step in a pathway could be understood and biologically active compounds for use as substrates for test enzymes were generated. Therefore, in recent years, a lack of understanding of the iridoid pathways has been a major obstacle. However, tecently developed rapid sequencing methods have opened up new opportunities. For example, transcriptome sequence data were obtained from C. rosesplants and cellular extract cultures. This method is effective in the EU Smart Cell Project, which aims to link transcriptional tumors, protective proteins, and microbiome data under a variety of conditions, select genetic markers, and then overexpress and test the putative activity of the encoded protein. Secoyid approach.36.34]. The identified genes can also be silenced, and the components can be determined by analyzing the intermediate genes found in plants. DeLuca et al. used gene sequencing databases for medicinal genomes and plant proteomics. ${ }^{39,41}$ In addition to elucidating the TIA pathway, its cellular and subcellular locations have already been reported. The main site of expression of the gene in the MEP pathway (such as DXR, DXS, MECS, HDS, and IDI) $)^{24,43}$ are endothelium-associated parenchymal cells (IPAP) around the mother pulp or in the armpit. The upper part of the connective tissue of the leaf. In situ hybridization RNA has been shown to be found in IPAP transcripts, such as GES, IS, 8HGO, IO, 7DLGT, 7DLH, and G80, such as the MEP gene. ${ }^{37-40}$ Such finding indicates that the roradic acid produced by these cells is transported, for example, to the epidermal cells, where the enzyme LAMT mechanizes roradic acid. In the epidermis, the secologanin synthase (SLS) gene product that provides CYP72A1 also 
has the highest possible expression, catalyzing the formation of the final oxidation step of iridoid glycosides. ${ }^{36,44,45}$ In situ RNA hybridization revealed that GES, IS, 8HGO, IO, 7DLGT, 7DLH, and $\mathrm{G} 80$ transcripts and genes involved in the MEP pathway were found in IPAP. ${ }^{37-40}$ Such finding indicates that the lognic acid produced by these cells is transported to epithelial tissue cells, such as LAMT enzyme-mechanized lognic acid. In this epidermis, the unique secologanin synthase (SLS) gene that provides CYP72A1 also has the highest possible expression, catalyzing the final oxidation step to form iridoid glycosides. ${ }^{36,44,45}$ Lognic acid is a transportable precursor that can move from IPAP to the epidermis, which means that this transport is a key bio-rate controller for flux in the production of secologanin. It also elaborates on the subcellular compartmentalization of the MEP secoiridoid pathway. The plastid contains the MEP pathway. ${ }^{16}$ HDS was detected in thylakoid-free bundles filled with a long matrix from plastids. ${ }^{46}$ IDI is located in the chloroplast, mitochondrial membrane, and peroxisome in $C$. roseus cells and is fused with green fluorescent protein (GFP). ${ }^{29}$ CrGPPS. $\mathrm{SSu}$ is a plastid, and CrGPPS is a mitochondrial function that depends on the location of GFP. ${ }^{31}$ GES was found in the matrix and plastid matrix after the transient transformation. Rose cells with yellow fluorescent protein fusion pattern. ${ }^{37}$ The endoplasmic reticulum (ER) has been clearly identified as the location of $\mathrm{G} 80 .^{46}$

Using fluorescent protein fusion and bimolecular fluorescence complementation tests, IS was shown to be completely localized in the cytoplasm. ${ }^{38}$ Using the fusion of green fluorescent tagged protein (GFP) in C. roseus cells and the mCherry marker, IO and 7DLH were found to be related to ER, while $8 \mathrm{HGO}$ and 7DLGT are soluble proteins found in the cytoplasm and the nucleus. ${ }^{40}$ In the cytosol, LAMT forms a homodimer, whereas SLS binds to the ER through an N-terminal helical loop. ${ }^{47}$ This is consistent with the hypothesis that ganic acid is a transport intermediate, which is methylated in the cytoplasmic compartment and then further oxidized in the ER to produce secologanin during the uptake of epidermal cells. These findings indicate a possible mechanism for the export of GPP from the plastid chromosome to the ER and the cytoplasmic binding enzymatic reaction to produce iridoids through the matrix. The Monoindole alkaloid biosynthesis and its localization

The MIA class in a heavily branched pathway is supported by specific transcription factors. ${ }^{78}$ Tryptophan is the precursor for all indole alkaloids that undergo decarboxylation to form tryptamine. The MIA pathway starts with the Mannich reaction ${ }^{77}$ of tryptamine and secologanin, which yields strictosidine, a key substrate produced by STR, from which the metabolic ions are then guided into various TIA biosynthetic pathways. ${ }^{5}$ Although STR is produced by a single gene, $C$. roseus has at least seven isotypes, all of which are usually the result of post-translational changes, primarily glycosylation, such as STR cinchona. ${ }^{48-50}$ Strictosidine undergoes deglycosylation in the presence of strictosidine-D glucosidase (SGD), ${ }^{79,80}$ which yields strictosidine aglycone, an unstable and highly reactive methanolamine that exists in several forms. ${ }^{50,51}$ Strictosidine aglycone endures three steps, and cathelamine is the precursor of ajmalicine and tetrahydroalstonine.$^{81}$ Cathenamine serves as the connection point between two different types of TIAs: 1) cathenamine is reduced to ajmalicine and then to serpentine by alstonine synthase, ${ }^{82} 2$ ) the imine salt of catechamine and the cofactor NADPH are converted to four by tetrahydroalstonine. Hydrocarbon, which then becomes oxidized to alanine. Reversible conversion to 4.21 dehydrogenated geissoschizine, which can form vinblastine and vincristine through the madanine stem. The biosynthetic pathway of Vendolin is well known, and it begins with tabersonine. There are 7 enzymatic catalytic steps in this process: 1) tabersonine 16-hydroxylasetabersonine 16-hydroxylase (T16H) results in 16 -hydroxy tabersonine ${ }^{83} ; 2$ ) tabersonine 16-0-methyltransferase (160MT) results in 16-methoxy hydroxytabersonin ${ }^{84}$; 3) tabersonine 3-oxygenase results in (3R)-3-Hydroxy 16-methoxy -1,2-dide hydro 2,3 - dihydrotabersonine ${ }^{85}$; 4) 3-hydroxy-1,2-didehydro-2,3-dihydrotabersonine reductase results in 16-methoxy-2,3-dihydro-3hydroxytabersonine ${ }^{85}$; 5) 3-hydroxy-16-methoxy2,3-dihydrotabersonine $\mathrm{N}$-methyltransferase results in Desacetoxyvindoline ${ }^{84}$; 6) deacetoxyvindoline 4-hydroxylase (D4H) is hydroxylated at the 4-position of deacetyl 
Vindoline ${ }^{86}$; and 7) deacetylvindoline- O- acetyl transferase (DAT) converts to acetylate deacetyl vindoline to form vindoline. ${ }^{87}$ Except for the unidentified hydroxylase, the genes for the other five enzymes have been cloned and described. Furthermore, T16H and SGD mRNA have been found to be more common in the epidermis. ${ }^{52,25}$ The anterior and posterior epidermal cell extracts were found to have the highest 160MT activity, while the whole leaf extract were found to have the highest NMT activity, indicating that it was found in the chloroplast thylakoid. Records (STR and MAT) exist in prodermal and cortical cells near the apical root meristem in underground cells. ${ }^{53,54}$ In short-term conversion attempts, the STRGFP signal occurs in the vacuole. ${ }^{55}$ STR is designated as a vacuole through the ER to the Golgi to the vacuole route. SGD is a dichotomous NLS centered on the nucleus and tends to multimerize in this compartment of the cell. ${ }^{56}$ In transient transformation experiments, STR GFP signals are generated in vacuoles. ${ }^{55}$ The STR is directed to the vacuole through the ER to the Golgi to the vacuole pathway. SGD is a dichotomous NLS that targets the nucleus and tends to multimerize in this cell compartment. ${ }^{56}$ The subcellular location of the vindoline pathway is well known.

\section{The bisindole alkaloids and their localization}

The combination of vinblastine and vinblastine to form anhydrovinblastine is catalyzed by $3^{\prime}, 4$ ' anhydrovinblastine synthase (AVLBS), which belongs to the class III alkaline peroxidase family. ${ }^{57}$ Vinblastine is formed from dehydrated vinblastine or its imine ion and is then converted to vincristine. ${ }^{10}$ Peroxidase is a group of isozymes that exists in all plant species and is involved in many physiological functions. In $C$. roseus, novel peroxidase genes, including $\mathrm{CrPrx}, \mathrm{CrPrx3}$, and CrPrx4 have been cloned and reported. However, the enzymes and genes involved in the next two stages are poorly understood. In subcellular localization investigations, CrPrx1 is believed to localize to vacuoles ${ }^{57}$ while CrPrx is largely apoplast. ${ }^{58,59}$

Metabolic Bioengineering of The Pathway in $\boldsymbol{C}$. roseus

Metabolic bioengineering is a technique that uses gene transfer to alter metabolic pathways and metabolite synthesis. Metabolic engineering of TIA biosynthesis in roses has been utilized to boost the production of certain TIAs as knowledge of the TIA pathways and metabolic genes has increased. Different types of TIA pathway genes have been found to be overexpressed in rose cells, hairy roots, and plants, with varying impacts on TIA synthesis and the complete metabolome.

Table 1. The work of TIAs, their functions and the factory that produces them

\begin{tabular}{lll}
\hline Different alkaloids & Bioactivity & Plants/species \\
\hline Ajmalicine & Lipid-lowering activity & C. roseus \\
Camptothecine & Anticancerous drug & Camptotheca acuminate \\
Ellipticine & Antitumourogenic drug & Ochrosia elliptica \\
Emetine & Anti-protozoan & Carapichea ipecacuanha \\
Quinidine & Class I Almokalant mediator (la) in the heart system & Cinchona spp \\
Quinine & Antibacterial drug & Cinchona spp. \\
Rèscinamine & Anti-hypertension & Rauwolfia spp. \\
Reserpine & Antipsychotic-agent, Antihypertensive & Rauwolfia serpentina \\
Serpentine & Soporific acceleration drug & C. roseus \\
Strychnene & Mutagen on mouse and use ib homeopathic & Strychnosnux-vomica \\
Toxiferine & Tubocurarine toxin, muscle relaxant & Strychnostoxifera \\
Vinblastine, & Antitumor & C. roseus \\
Vincristine & Anticancer activity & C. roseus \\
Vincamine & Peripheral vasodilators increase blood flow to nerve & Vinca minor \\
& growth in the brain & \\
Yohimbine & Mild monoamine oxidase inhibitors with aphrodisiac & Corynante Yohimbe \\
& and aphrodisiac effects &
\end{tabular}




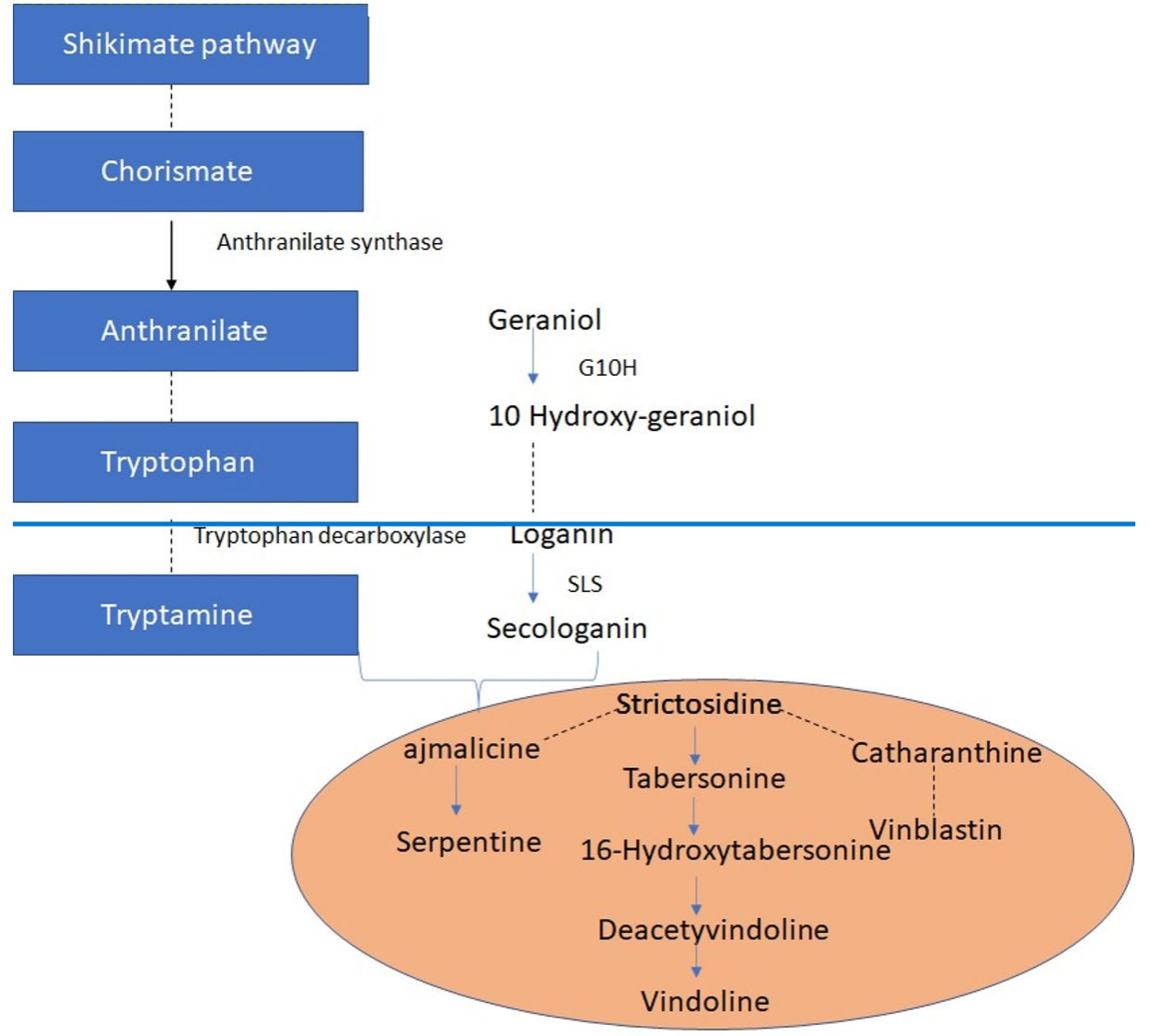

Fig. 1. TIA metabolic pathway in C.roseus. Single enzymatic reactions are indicated by solid arrow, while numerous enzymatic conversions are indicated by dashed arrows

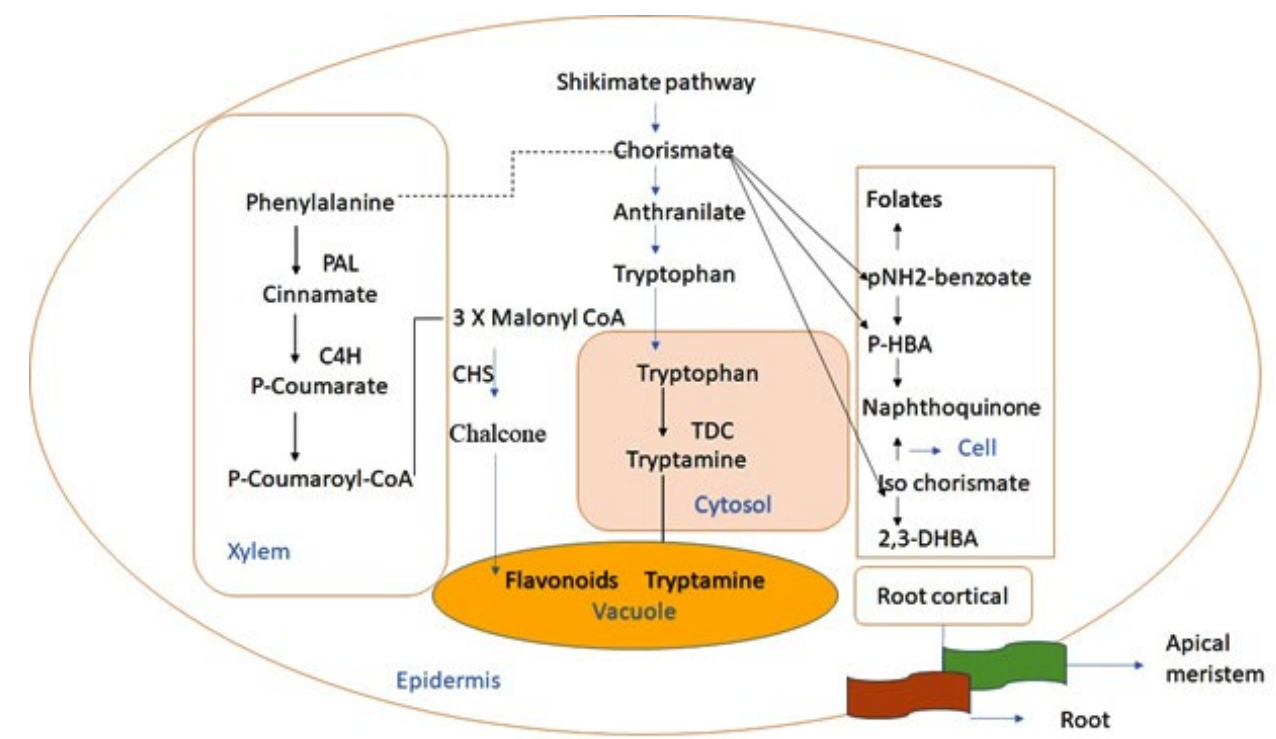

Fig. 2. The shikimate chorismateindole pathway and its division at the intercellular and subcellur levels. The solid arrow represent a one step reaction; broken arrows represent multiple reactions or unidentified reactions 


\section{Omics Tool Plus System Biology}

To predict gene function, researchers use a combination of omics to characterize the coordination of complex interactions with plant metabolic networks in the course of biological processes from the perspective of systems biology. ${ }^{60}$ A combination of transcriptomics, metabolomics, and unidirectional methods can represent a potential filter network of genetic metabolites. ${ }^{61}$ From a biological point of view, integration with other omics is used to predict gene function and biological processes and characterize complex interactions and coordination of plant metabolic networks..$^{60}$ The combination of one-way approaches, such as transcription and metabolism, can uncover putative gene metabolite networks, ${ }^{61}$ identify candidate genes for specific metabolic pathways, ${ }^{62}$ and examine genetic function by overexpression. ${ }^{61,63}$ Several omics techniques are integrated to predict genetic function and analyze the complex interactions and coordination of plant metabolic networks in biological processes used in biological systems. ${ }^{60}$ The combination of non-targeted techniques, such as transcription and metabolism, can uncover possible networks of gene metabolism ${ }^{61}$ and screen promising genes for certain biosynthetic processes ${ }^{62} \mathrm{~A}$ remarkable example is the study of the circadian clock mechanism in Arabidopsis thaliana ${ }^{60}$ The biological importance of pseudo-response regulators (PRR 9, 7, and 5) in Arabidopsis was studied using two arrhythmogenic plants, an overpressurized Cicardian Clock Associated 1 strain (CCA1ox), and triple mutations PRR 9, 7, and 5 (d975). Metabolic profiling revealed that $\mathrm{d} 975$ exhibited a significant increase in TCA cycle intermediates, while CCA1ox exhibited fewer changes in primary metabolism. The metabolic profile of the induced cell culture of $C$. roseus was associated with the genome-wide transcriptional profile of the amplified fragment length polymorphism of cDNA to generate a range of session tags. Codes and metabolites linked to the TIA of $C$. roseus. ${ }^{64} \mathrm{~A}$ hitherto unknown network of genes and metabolites was discovered by comparing the expression profiles of 417 gene markers with the cumulative form of 178 metabolite peaks. According to these networks, many branches of the biosynthesis of terpene indole alkaloids and many other metabolic processes are controlled by different hormones. These networks have also led to the discovery of genes and metabolites involved in the production of indole terpenoid alkaloids. Therefore, the combination of multiple omics technologies is expected to help identify key regulatory genes and characterize pathway interactions in different processes, to ultimately reveal systemic coordination and communication in the metabolic network of plants.

\section{CONCLUSION AND FUTURE ASPECTS}

Based on current knowledge of $C$. roseus, we sought to summarize how various types of small molecules affect the TIA biosynthetic pathway. In recent decades, due to the development of new technologies in molecular biology and molecular biochemistry, great progress in understanding TIA biosynthesis and regulation in $C$. roseus has been made. However, there are still many areas that have not yet been investigated. Further studies are needed to identify genes, enzymes, structural intermediates, and genes involved in pathway control. This information is necessary for the development of transgenic plants, plant cells, and microorganisms to commercialize extremely valuable dimeric alkaloids.

Plant genetic manipulation, plant cell culture, and microorganisms have failed to produce TIA at an economically viable cost. Further, this route is more complicated than a series of enzymatic steps. The compartmentalization of plant pathways includes many cell types. One of the challenging questions is whether compartments exist in these cells. Due to the complexity of the pathway, cell suspension culture cannot be used to assess all stages. The logistics for successful biosynthesis must be established, which means that all intermediates, as well as all cofactors and ATP needed for biochemical reactions, must exist at the right time, in the right quantity, and in the right place.

The TIA pathway does not exist in isolation throughout the plant's metabolic network, but instead crosses and interacts with other branching pathways, which implies that it is part of a complex matrix. Accordingly, the total amount of carbon that plants contain for TIA biosynthesis must be elucidated. Ultimately, a systems biology approach is needed to address some of these challenges, which means that all omics are needed to identify 
the missing links in dynamic mapping of the TIA and biosynthetic system. The availability of the complete transcriptome sequence of a variety of indole-producing plants under various conditions will help identify the structural genes involved in these pathways. Regulatory genes can be more difficult to identify as the regulation of biosynthesis varies from species to species. Even within a single plant, the regulation between the TIA production areas and the specific cells that process different parts of the cascade can vary. Therefore, a unicellular method will become a key tool in deciphering the pathway, regulation, and physiological relevance of alkaloids in plants. Single-cell research is crucial in terms of compartments and transport, because the different types of cells involved are expected to have different modes of selective transporters that are used to absorb and extrude intermediates into various cell compartments and whole cells. For example, in a leaf, iridoid-producing cells are more likely to have transporters to squeeze out organic acids, whereas epidermal cells will have transporters to selectively receive this chemical. This transporter may not be present in nonalkaloid-producing cells, or may be replaced by a transporter that squeezes out the intermediate. Furthermore, the specific accumulation of alkaloids in specific cells requires specialized absorption and/or extrusion of alkaloids. A long and difficult journey still exists to gain a full understanding of TIA biosynthesis, which will be very useful for better understanding the metabolism of antioxidants in plants. Undoubtedly, such research will provide insights for the production of dimer alkaloids, similar to the discovery of the coupling method of vinblastine and ventolin ${ }^{65}$ resulting in the chemical production of dimers on an industrial scale. Secondary metabolites are unique among the many chemicals produced by gene expression owing to their wide range of applications, including drugs, fragrances and perfumes, dyes and pigments, pesticides, and food additives. Alkaloids are a class of low-molecular-weight nitrogencontaining organic molecules with heterocyclic structures, which account for a large proportion of these metabolites. They are particularly important due to their multiple biological activities, including therapeutic capabilities ${ }^{6}$ and their key ecological role. The biosynthesis and accumulation of alkaloids are properly regulated processes, according to several studies on alkaloidproducing plants. Among alkaloid plants, the indole monoterpene alkaloid plant is a rich source of numerous medications. Compounds, such as vinblastine and vincristine (anti-tumor), vincristine and vincristine (antihypertensive), and amaricine and amarine (anti-malarial drugs) are examples of such molecules. Extracting information from a large amount of information generated by high-throughput genomics technology requires powerful bioinformatics tools. Research using transcriptomes and expression profiles provides an opportunity to better understand plant metabolism and enable analyses of the formation of plant-derived drugs from $C$. roseus.

\section{ACKNOWLEDGMENTS}

None.

\section{CONFLICT OF INTEREST}

The authors declare that there is no conflict of interest.

\section{AUTHORS' CONTRIBUTION}

All authors listed have made a substantial, direct and intellectual contribution to the work, and approved it for publication.

\section{FUNDING}

None.

\section{DATA AVAILABILITY}

All datasets generated or analyzed during this study are included in the manuscript.

\section{ETHICS STATEMENT}

Not applicable.

\section{REFERENCES}

1. Zhu X, Zeng X, Sun C, Chen S. Biosynthetic pathway of terpenoid indole alkaloids in Catharanthus roseus. Front Med. 2014;8(3):285-293. doi: 10.1007/s11684014-0350-2

2. Zhao L, Sander GW, Shanks JV. Perspectives of the metabolic engineering of terpenoid indole alkaloids in Catharanthus roseus hairy roots. Adv Biochem Eng Biotechnol. 2013;134:23-54. doi: 10.1007/10_2013_182

3. Kutchan TM. Strictosidine: from alkaloid to enzyme to gene. Phytochemistry. 1993:32(3):493-506. doi: 10.1016/S0031-9422(00)95128-8 
4. Zhu J, Wang M, Wen W, Yu R. Biosynthesis and regulation of terpenoid indole alkaloids in Catharanthus roseus. Pharmacogn Rev. 2015;9(17):2428. doi: $10.4103 / 0973-7847.156323$

5. Pan Q, Mustafa NR, Tang K, Choi YH, Verpoorte R. Monoterpenoid indole alkaloids biosynthesis and its regulation in Catharanthus roseus: a literature review from genes to metabolites. Phytochem Rev. 2016;15(2):221-250. doi: 10.1007/s11101-015-9406-4

6. Hirata K, Miyamoto K, Miura Y. Catharanthus roseus L. (Periwinkle): Production of Vindoline and Catharanthine in Multiple Shoot Cultures. Biotechnology in Agriculture and Forestry. Medicinal and Aromatic Plants. VI. Springer-Verlag. 1994;26:4655. doi: 10.1007/978-3-642-57970-7_3

7. Van der Heijden R, Jacobs DI, Snoeijer W, Hallard $D$, Verpoorte R. The Catharanthus alkaloids: Pharmacognosy and biotechnology. Curr Med Chem 2004;11(5):607-628. doi: 10.2174/0929867043455846

8. Scott Al, Mizukami H, Lee SL. Characterization of a 5-methyltryptophan resistant strain of Catharanthus roseus cultured cells. Phytochemistry. 2004;18(5):795798. doi: 10.1016/0031-9422(79)80017-5

9. De Luca V, Laflamme P. The expanding universe of alkaloid biosynthesis. Curr Opin Plant Biol. 2001:4:225233. doi: 10.1016/S1369-5266(00)00165-5

10. Verpoorte $R$, van der Heijden R, Moreno PR. Biosynthesis of Terpenoid Indole Alkaloids in Catharanthus roseus Cells. Alkaloids: Chem Pharmacol. 1997;49:221-299. doi: 10.1016/S0099-9598(08)600176

11. Sharma A, Amin D, Sankaranarayanan A, Arora R, Mathur AK. Present status of Catharanthus roseus monoterpenoid indole alkaloids engineering in homoand hetero-logous systems. Biotechnology Letters. 2020:42(1):11-23. doi: 10.1007/s10529-019-02757-4

12. Grothe T, Kutchan TM, Zenk MH. Salutaridinol 7-o-acetyltransferase and derivatives thereof. Google Patents. 2002. https://patents.google.com/patent/ WO2002101052A3/en

13. Gongora-Castillo E, Fedewa G, Yeo Y, Chappell J, Penna DD, Buell CR. Genomic Approaches for Interrogating the Biochemistry of Medicinal Plant Species. Methods Enzymol. 2012;517:139-159. doi: 10.1016/B978-0-12404634-4.00007-3

14. Liu L-YD, Tseng $\mathrm{HI}$, Lin CP, et al. High-throughput transcriptome analysis of the leafy flower transition of Catharanthus roseus induced by peanut witches'broom phytoplasma infection. Plant Cell Physiol .2014;55(Suppl 1):942-957. doi: 10.1093/pcp/pcu029

15. Verma M, Ghangal R, Sharma R, Sinha A K, Jain M. Transcriptome Analysis of Catharanthus roseus for Gene Discovery and Expression Profiling. PLOS ONE. 2014;9(7):e103583. doi: 10.1371/journal. pone. 0103583

16. Roytrakul S, Verpoorte R. Role of vacuolar transporter proteins in plant secondary metabolism: Catharanthus roseus cell culture. Phytochem Rev. 2007;6(2):383-396. doi: 10.1007/s11101-006-9022-4

17. Verma P, Mathur AK, Srivastava A, Mathur A. Emerging Trends in research on spatial and temporal organization of terpenoid indole alkaloid pathway in
Catharanthus roseus: a literature update. Protoplasma. 2012;249:255-268. doi: 10.1007/s00709-011-0291-4

18. Herrmann KM, Weaver LM. The shikimate pathway. Ann Rev Plant Boil. 1999;50:473-503. doi: 10.1146/ annurev.arplant.50.1.473

19. Mustafa NR, Verpoorte R. Phenolic compounds in Catharanthus roseus. Phytochem Rev. 2007;6(2):243258. doi: 10.1007/s11101-006-9039-8

20. Poulsen C, Bongaerts RJ, Verpoorte R. Purification and characterization of anthranilate synthase from Catharanthus roseus. Eur J Biochem. 1993;212(2):431440. doi: 10.1111/j.1432-1033.1993.tb17679.x

21. Luttik MA, Vuralhan Z, Suir E, Braus GH, Pronk JT, Daran JM. Alleviation of feedback inhibition in Saccharomyces cerevisiae aromatic amino acid biosynthesis: quantification of metabolic impact. Metabolic Engineering. 2008;10(3-4):141-153. doi: 10.1016/j.ymben.2008.02.002

22. Radwanski ER, Last RL. Tryptophan biosynthesis and metabolism: biochemical and molecular genetics. Plant Cell. 1995;7(7):921-934. doi: 10.2307/3870047

23. Reyes-Prieto A, Moustafa A. Plastid-localized amino acid biosynthetic pathways of Plantae are predominantly composed of non-cyanobacterial enzymes. Scientific Reports. 2012;2(1):955. doi: 10.1038/srep00955

24. Mahroug S, Courdavault V, Thiersault M, St-Pierre $B$, Burlat V. Epidermis is a pivotal site of at least four secondary metabolic pathways in Catharanthus roseus aerial organs. Planta. 2006;223:(6)1191-1200. doi: 10.1007/s00425-005-0167-y

25. Murata J, Roepke J, Gordon H, De-Luca V. The leaf epidermome of Catharanthus roseus reveals its biochemical specialization. Plant Cell. 2008;20(3):524542. doi: 10.1105/tpc.107.056630

26. Buhaescu I, Izzedine H. Mevalonate pathway: a review of clinical and therapeutical implications. Clin Biochem. 2007;40(9-10):575-584. doi: 10.1016/j. clinbiochem.2007.03.016

27. Lange BM, Croteau R. Isopentenyl diphosphate biosynthesis via a mevalonate-independent pathway: isopentenyl monophosphate kinase catalyzes the terminal enzymatic step. Proc Natl Acad Sci USA. 1999;96(24):13714-13719. doi: 10.1073/ pnas.96.24.13714

28. El-Sayed M, Verpoorte R. Catharanthus terpenoid indole alkaloids: biosynthesis and regulation. Phytochem Rev. 2007;6:277-305. doi: 10.1007/s11101006-9047-8

29. Guirimand G, Guihur A, Phillips MA, et al. A single gene encodes isopentenyl diphosphate isomerase isoforms targeted to plastids, mitochondria and peroxisomes in Catharanthus roseus. Plant Mol Biol. 2012;79(45):443-459. doi: 10.1007/s11103-012-9923-0

30. Hedhili S, Courdavault V, Giglioli-Guivarc'h N, Gantet P. Regulation of the terpene moiety biosynthesis of Catharanthus roseus terpene indole alkaloids. Phytochem Rev. 2007;6(2):341-351. doi: 10.1007/ s11101-006-9021-5

31. Rai A, Smita SS, Singh AK, Shanker K, Nagegowda DA. Heteromeric and homomeric geranyl diphosphate synthases from Catharanthus roseus and their role 
in monoterpenoid indole alkaloid biosynthesis. $\mathrm{Mol}$ Plant. 2013;6(5):1531-1549. doi: 10.1093/mp/sst058

32. Collu G, Unver N, Peltenburg-Looman AM, Heijden RVD, Verpoorte R, Memelink J. Geraniol 10-hydroxylase, a cytochrome P450 enzyme involved in terpenoid indole alkaloid biosynthesis. Febs Lett. 2001;508(2):215-220. doi: 10.1016/S0014-5793(01)03045-9

33. Hofer R, Dong L, Andre F, et al. Geraniol hydroxylase and hydroxygeraniol oxidase activities of the CYP76 family of cytochrome P450 enzymes and potential for engineering the early steps of the (seco) iridoid pathway. Metabolic Engineering. 2013;20:221-232. doi: 10.1016/j.ymben.2013.08.001

34. Miettinen, Karel, Lemeng Dong, Nicolas Navrot, Thomas Schneider, Vincent Burlat, Jacob Pollier, Lotte Woittiez et al. "The seco-iridoid pathway from Catharanthus roseus." Nature communications 5, no. 1 (2014): 1-12. doi: $10.1038 /$ ncomms 4606

35. Luijendijk TJC, Stevens LH, Verpoorte R. Purification and characterisation of strictosidine $\beta$-D-glucosidase from Catharanthus roseus cell suspension cultures. Plant Physiol Biochem. 1998;36:419-425. doi: 10.1016/ S0981-9428(98)80205-2

36. Irmler S, Schroder G, St-Pierre B, et al. Indole alkaloid biosynthesis in Catharanthus roseus: new enzyme activities and identification of cytochrome P450 CYP72A1 as secologanin synthase. Plant J. $2000 ; 24(6): 797-804$. doi: $10.1046 /$ j.1365313x.2000.00922.x

37. Simkin AJ, Miettinen K, Claudel P, et al . Characterization of the plastidial geraniol synthase from Madagascar periwinkle which initiates the monoterpenoid branch of the alkaloid pathway in internal phloem associated parenchyma. Phytochemistry. 2013;85:36-43. doi: 10.1016/j.phytochem.2012.09.014

38. Geu-Flores F, Sherden NH, Courdavault V, Burlat V, Glenn WS, et al. An alternative route to cyclic terpenes by reductive cyclization in iridoid biosynthesis. Nature. 2012;492(7427):138-142. doi: 10.1038/nature11692

39. Asada K, Salim V, Masada-Atsumi S, et al. A 7-Deoxyloganetic Acid Glucosyltransferase Contributes a Key Step in Secologanin Biosynthesis in Madagascar Periwinkle. Plant Cell. 2013;25(10):4123-4134. doi: $10.1105 /$ tpc.113.115154

40. Miettinen K. Elucidation of the secoiridoid pathway in Catharanthus roseus. PhD Thesis, Leiden University, The Netherlands. 2013.

41. Salim V, Yu F, Altarejos J, De Luca V. Virus-induced gene silencing identifies Catharanthus roseus 7-deoxyloganic acid-7-hydroxylase, a step in iridoid and monoterpene indole alkaloid biosynthesis. Plant J. 2013;76(5):754-765. doi: 10.1111/tpj.12330

42. Dong L, Miettinen K, Goedbloed $M$, et al. Characterization of two geraniol synthases from Valeriana officinalis and Lippia dulcis: Similar activity but difference in subcellular localization. Metab Eng. 2013;20:198-211. doi: 10.1016/j.ymben.2013.09.002

43. Burlat V, Oudin A, Courtois M, Rideau M, St-Pierre B. Coexpression of three MEP pathway genes and geraniol 10-hydroxylase in internal phloem parenchyma of Catharanthus roseus implicates multicellular translocation of intermediates during the biosynthesis of monoterpene indole alkaloids and isoprenoidderived primary metabolites. Plant J. 2004;38(1):131141. doi: 10.1111/j.1365-313X.2004.02030.x

44. St-Pierre B, Vazquez-Flota FA, De-Luca V. Multicellular compartmentation of Catharanthus roseus alkaloid biosynthesis predicts intercellular translocation of a pathway intermediate. Plant Cell. 1999;11(5):887-900. doi: $10.1105 /$ tpc.11.5.887

45. Yamamoto H, Katano N, Ooi A, Inoue K. Secologanin synthase which catalyzes the oxidative cleavage of loganin into secologanin is a cytochrome P450. Phytochemistry. 2000;53(1):7-12. doi: 10.1016/S00319422(99)00471-9

46. Guirimand G, Burlat V, Oudin A, Lanoue A, St-Pierre $B$, Courdavault V. Optimization of the transient transformation of Catharanthus roseuscells by particle bombardment and its application to the subcellular localization of hydroxymethylbutenyl 4-diphosphate synthase and geraniol 10-hydroxylase. Plant Cell Rep. 2009;28(8):1215-1234. doi: 10.1007/s00299-009$0722-2$

47. Guirimand G, Guihur A, Ginis O, et al. The subcellular organization of strictosidine biosynthesis in Catharanthus roseus epidermis highlights several trans-tonoplast translocations of intermediate metabolites. FEBS J. 2011a;278(5):749-763. doi: 10.1111/j.1742-4658.2010.07994.x

48. Stevens LH, Giroud C, Pennings EJ, Verpoorte R. Purification and characterization of strictosidine synthase from a suspension culture of Cinchona robusta. Phytochemistry. 1993;33(1):99-106. doi: 10.1016/0031-9422(93)85403-E

49. McKnight T, Roessner C, Devagupta R, Scott A, Nessler C. Nucleotide sequence of a cDNA encoding the vacuolar protein strictosidine synthase from Catharanthus roseus. Nucl Acids Res. 1990;18(16):4939. doi: $10.1093 /$ nar/18.16.4939

50. Barleben L, Panjikar S, Ruppert M, Koepke J, Stockigt J. Molecular architecture of strictosidine glucosidase: the gateway to the biosynthesis of the monoterpenoid indole alkaloid family. The Plant Cell. 2007;19(9):28862897. doi: $10.1105 /$ tpc. 106.045682

51. Geerlings A, Ibanez MM-L, Memelink J, van der Heijden $R$, Verpoorte R. Molecular cloning and analysis of strictosidine $\beta$-D-glucosidase, an enzyme in terpenoid indole alkaloid biosynthesis in Catharanthus roseus. J Biol Chem. 2000;275:(5)3051-3056. doi: 10.1074/ jbc.275.5.3051

52. Murata J, De-Luca V. Localization of tabersonine 16-hydroxylase and 16-OH tabersonine-16-0methyltransferase to leaf epidermal cells defines them as a major site of precursor biosynthesis in the vindoline pathway in Catharanthus roseus. Plant J. 2005;44(4):581-594. doi: 10.1111/j.1365313X.2005.02557.x

53. Laflamme P, St-Pierre B, De Luca V. Molecular and biochemical analysis of a Madagascar periwinkle root-specific minovincinine-19-hydroxy-0acetyltransferase. Plant Physiol. 2001;125(1):189-198. doi: 10.1104/pp.125.1.189

54. Moreno-Valenzuela OA, Minero-Garcia Y, MayerGeraldo E, Carbajal E, Loyola-Vargas VM. Increase in 
the indole alkaloid production and its excretion into the culture medium by calcium antagonists in Catharanthus roseus hairy roots. Biotechnol Lett. 2003;25(16):13451349. doi: 10.1023/A:1024988605769

55. Guirimand G, Courdavault V, St-Pierre B, Burlat V. Biosynthesis and regulation of alkaloids. Plant Dev Biol Biotechnol Perspect. 2010;139-160. doi: 10.1007/9783-642-04670-4_8

56. Guirimand G, Courdavault V, Lanoue A, et al. Strictosidine activation in Apocynaceae: towards a "nuclear time bomb". BMC Plant Biol. 2010;10:182. doi: 10.1186/1471-2229-10-182

57. Costa MMR, Hilliou F, Duarte P, et al. Molecular cloning and characterization of a vacuolar class III peroxidase involved in the metabolism of anticancer alkaloids in Catharanthus roseus. Plant Physiol. 2008;146(2):323324. doi: 10.1104/pp.107.107060

58. Kumar S, Dutta A, Sinha AK, Sen J. Cloning, characterization and localization of a novel basic peroxidase gene from Catharanthus roseus. FEBS J. 2007;274(5):1290-1303. doi: 10.1111/j.17424658.2007.05677.x

59. Kumar S, Jaggi M, Sinha AK. Ectopic overexpression of vacuolar and apoplastic Catharanthus roseus peroxidases confers differential tolerance to salt and dehydration stress in transgenic tobacco. Protoplasma. 2012;249(2):423-432. doi: 10.1007/s00709-011-02941

60. Fukushima A, Kusano M, Redestig H, Arita M, Saito K. Integrated omics approaches in plant systems biology. Curr Opin Chem Biol. 2009;13(5-6):532-538. doi: 10.1016/j.cbpa.2009.09.022

61. Urbanczyk-Wochniak E, Luedemann A, Kopka J, et al. Parallel analysis of transcript and metabolic profiles: a new approach in systems biology. Embo Rep. 2003;4(10):989-993. doi: 10.1038/sj.embor.embor944

62. Okazaki Y, Shimojima M, Sawada Y, et al. A chloroplastic UDP-glucose pyrophosphorylase from Arabidopsis is the committed enzyme for the first step of sulfolipid biosynthesis. Plant Cell. 2009;21(3):892-909. doi: 10.1105/tpc.108.063925

63. Lackman P, Gonzalez-Guzman M, Tilleman S, Carqueijeiro I, Perez AC. Jasmonate signaling involves the abscisic acid receptor PYL4 to regulate metabolic reprogramming in Arabidopsis and tobacco. Proc Natl Acad Sci USA. 2011;108(14):5891-5896. doi: 10.1073/ pnas.1103010108

64. Rischer H, Oresic M, Seppanen-Laakso T, et al. Geneto-metabolite networks for terpenoid indole alkaloid biosynthesis in Catharanthus roseus cells. Proc Natl Acad Sci USA. 2006;103(14):5614-5619. doi: 10.1073/ pnas.0601027103

65. Goodbody AE, Endo T, Vukovic J, Kutney JP, Choi LS, Misawa M. Enzymic coupling of catharanthine and vindoline to form $3^{\prime}, 4^{\prime}$-anhydrovinblastine by horseradish peroxidase. Planta Med. 1988;54(2):136140. doi: 10.1055/s-2006-962371

66. Van der Fits L, Memelink J. ORCA3, a jasmonateresponsive transcriptional regulator of plant primary and secondary metabolism. Science. 2000;289(5477):295297. doi: 10.1126/science.289.5477.295

67. Li CY, Leopold AL, Sander GW, Shanks JV, Zhao L, Gibson
SI. The ORCA2 transcription factor plays a key role in regulation of the terpenoid indole alkaloid pathway. BMC Plant Biology. 2013:13:155. doi: 10.1186/14712229-13-155

68. Menke FL, Champion A, Kijne JW, Memelink J. A novel jasmonate- and elicitor-responsive element in the periwinkle secondary metabolite biosynthetic gene Str interacts with a jasmonate- and elicitor-inducible $\mathrm{AP}_{2}$-domain transcription factor, $\mathrm{ORCA}_{2}$. The $E M B O$ Journal. 1999:18(16):4455-4463. doi: 10.1093/ emboj/18.16.4455

69. Suttipanta N, Pattanaik S, Kulshrestha M, Patra B, Singh SK, Yuan L. The transcription factor CrWRKY1 positively regulates the terpenoid indole alkaloid biosynthesis in Catharanthus roseus. Plant Physiol. 2011;157(4):20812093. doi: 10.1104/pp.111.181834

70. Li CY, Leopold AL, Sander GW, Shanks JV, Zhao L, Gibson SI. CrBPF1 overexpression alters transcript levels of terpenoid indole alkaloid biosynthetic and regulatory genes. Front Plant Sci. 2015;6:818. doi: 10.3389/ fpls.2015.00818

71. Sazegari S, Niazi A, Shahriari-Ahmadi F, Moshtaghi N, Ghasemi Y. CrMYC1 transcription factor overexpression promotes the production of low abundance terpenoid indole alkaloids in Catharanthus roseus. Plant Omics. 2018:11(1):30-36. doi: 10.21475/poj.11.01.18. pne1020

72. Paul P, Singh SK, Patra B, Sui X, Pattanaik S, Yuan L. A differentially regulated AP2/ERF transcription factor gene cluster acts downstream of a MAP kinase cascade to modulate terpenoid indole alkaloid biosynthesis in Catharanthus roseus.New Phytol. 2017;213(3):11071123. doi: 10.1111/nph.14252

73. Li CY, Gibson SI. Repression of ZCT1, ZCT2 and ZCT3 affects expression of terpenoid indole alkaloid biosynthetic and regulatory genes. Peer J. 2021;9(392):e11624. doi: 10.7717/peerj.11624

74. Pauw B, Hilliou FAO, Martin VS, et al. Zinc finger proteins act as transcriptional repressors of alkaloid biosynthesis genes in Catharanthus roseus. J Biol Chem. 2004;279:52940-52948. doi: 10.1074/jbc. M404391200

75. Siberil Y, Benhamron S, Memelink J, et al. Catharanthus roseus G-box binding factors 1 and 2 act as repressors of strictosidine synthase gene expression in cell cultures. Plant Mol Biol. 2001;45(4):477-488. doi: 10.1023/A:1010650906695

76. Sharma A, Verma P, Mathur A, Mathur AK. Genetic engineering approach using early Vinca alkaloid biosynthesis genes led to increased tryptamine and terpenoid indole alkaloids biosynthesis in differentiating cultures of Catharanthus roseus. Protoplasma. 2017:255(1):425-435. doi: 10.1007/ s00709-017-1151-7

77. Mannich C, Krosche W. Ueberein Kondensationsprodukt aus Formaldehyd, Ammoniak und Antipyrin. Archiv der Pharmazie. 1912:250(1):647-667. doi: 10.1002/ ardp.19122500151

78. Colinas M, Pollier J, Vaneechoutte D, et al. Subfunctionalization of paralog transcription factors contributes to regulation of alkaloid pathway branch choice in Catharanthus roseus. Front Plant Sci. 
2021;12:687406. doi: 10.3389/fpls.2021.687406

79. Geerlings A, Ibanez MM, Memelink J, van Der Heijden $R$, Verpoorte R. Molecular cloning and analysis of strictosidine beta-D-glucosidase, an enzyme in terpenoid indole alkaloid biosynthesis in Catharanthus roseus. J Biol Chem. 2000;275(5):3051-3056. doi: 10.1074/jbc.275.5.3051

80. Hemscheidt T, Zenk MH. Glucosidases involved in indole alkaloid biosynthesis of Catharanthus cell cultures. FEBS Lett. 1980:110(2):187-191. doi: 10.1016/0014-5793(80)80069-X

81. Stavrinides A, Tatsis EC, Caputi L,et al. Structural investigation of heteroyohimbine alkaloid synthesis reveals active site elements that control stereoselectivity. Nat Commun. 2016:7:12116. doi: $10.1038 /$ ncomms12116

82. Miettinen K, Dong L, Navrot N, et al. The seco-iridoid pathway from Catharanthus roseus. Nat Commun. 2014:5:4175. doi: 10.1038/ncomms5175

83. St-Pierre B, De Luca V. A Cytochrome P-450 Monooxygenase Catalyzes the First Step in the Conversion of Tabersonine to Vindoline in Catharanthus roseus. Plant Physiol. 1995:109(1):131-
139. doi: $10.1104 / p p .109 .1 .131$

84. De Luca V, Balsevich J, Tyler RT, Eilert U, Panchuk $B D$, Kurz WGW. Biosynthesis of indole alkaloids developmental regulation of the biosynthetic-pathway from tabersonine to vindoline in Catharanthus roseus. J Plant Physiol. 1986:125(1-2):147-156. doi: 10.1016/ S0176-1617(86)80252-8

85. Qu Y, Easson ML, Froese J, Simionescu R, Hudlicky $\mathrm{T}$, De Luca V. Completion of the seven-step pathway from tabersonine to the anticancer drug precursor vindoline and its assembly in yeast. Proc Natl Acad Sci U S A. 2015:112(19):6224-6229. doi: 10.1073/ pnas.1501821112

86. De Carolis E, Chan F, Balsevich J, De Luca V. Isolation and Characterization of a 2-Oxoglutarate Dependent Dioxygenase Involved in the Second-to-Last Step in Vindoline Biosynthesis. Plant Physiol. 1990:94(3):13231329. doi: $10.1104 /$ pp. 94.3 .1323

87. Fahn W, Gundlach H, Deus-Neumann B, Stockigt J. Late enzymes of vindoline biosynthesis. Acetyl-CoA: 17-O-deacetylvindoline 17-0-acetyl-transferase. Plant Cell Rep. 1985:4(6):333-336. doi: 10.1007/BF00269892 